\title{
Inmunización con vacuna conjugada de neumococo y otras vacunas recomendadas en pacientes pediátricos de alto riesgo
}

\author{
Immunization with pneumococcal conjugate vaccine and other \\ recommended vaccines in high-risk pediatric patients
}

\author{
Federico Javier Ortiz-Ibarra,* Luis Xóchihua Díaz, ${ }^{\ddagger}$ Napoleón González Saldaña, ${ }^{\S}$ \\ Juana del Carmen Chacón Sánchez," Antonio Luévanos Velázquez," Francisco Javier Otero Mendoza,** \\ Mariana Merlo Palomera, ${ }^{\ddagger}$ Brandon Ortiz-Casas, ${ }^{\S \S}$ Nideshda Ramírez Uribe, ${ }^{\text {,q }}$ Enrique Rodríguez Barragán, ${ }^{* * *}$ \\ Guillermo Hideo Wakida Kusunoki, ${ }^{\# \ddagger}$ Germán Camacho-Moreno, ${ }^{\S \S}$ Francisco Espinosa Rosales ${ }^{\text {ฯ }}$

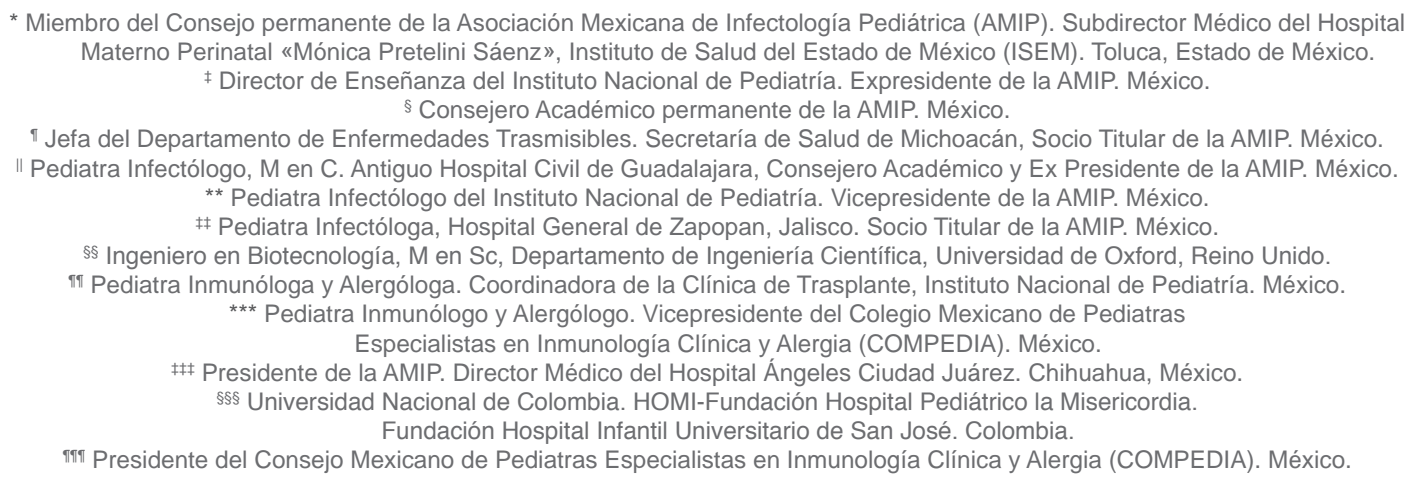

\section{RESUMEN}

Los pacientes inmunocomprometidos son el grupo más afectado y con mayores tasas de mortalidad asociada a infecciones invasivas, por lo que la vacunación del paciente inmunocomprometido desempeña un papel fundamental en la mejoría de su sobrevida y calidad de vida. Dado que la seguridad y eficacia de estos inmunógenos va a depender del tipo y grado de inmunosupresión individual, es importante conocer cuáles son las vacunas indicadas, y cuáles las contraindicadas que pueden tener un riesgo para estos pacientes. El presente consenso tuvo como objetivo analizar y discutir el impacto de la vacunación contra neumococo y otras vacunas recomendadas en la actualidad para el paciente pediátrico de alto riesgo. Material y métodos: Se utilizó el modelo de consenso académico, donde se discutió la literatura

\section{ABSTRACT}

Immunocompromised patients are of the most affected groups, with the highest mortality rates associated with invasive infections. Due to this, vaccination of immunocompromised patients plays a critical role in improving both survival and quality of life. Since the safety and efficacy of these immunogens will depend on the type and degree of individual immunosuppression, it is relevant to know which are the most indicated vaccines, and which are the contraindicated ones that may present a health risk for these patients. Therefore, this consensus aimed to analyze and discuss the impact of pneumococcal vaccination, as well as other vaccines currently recommended for high-risk pediatric patients. Material and methods: An academic consensus model was used, where the available clinical literature was systematically discussed.

Citar como: Ortiz-lbarra FJ, Xóchihua DL, González SN, Chacón SJC, Luévanos VA, Otero MFJ et al. Inmunización con vacuna conjugada de neumococo y otras vacunas recomendadas en pacientes pediátricos de alto riesgo. Rev Latin Infect Pediatr. $2021 ; 34$ (4): 162-176. https://dx.doi.org/10.35366/102965

Recibido: 24-08-2021. Aceptado: 22-10-2021.

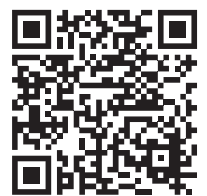


Rev Latin Infect Pediatr. 2021; 34 (4): 162-176

científica disponible. Para ello, se usó la guía Lineamientos para la elaboración de consensos y las recomendaciones y criterios útiles para la lectura crítica de los artículos seleccionados de la iniciativa europea AGREE. Para la conformación del grupo participante se integraron equipos mixtos y representativos en los que participaron pediatras infectólogos, miembros y pediatras con especialidad en inmunología y alergia así como pediatras del sector privado. Se realizó una búsqueda activa de la literatura dentro del periodo de enero de 2015 a agosto de 2021. Resultados: Se tomaron 10 aspectos relevantes en forma de preguntas, para las cuales se establecieron recomendaciones con base en los artículos críticamente leídos, presentando los niveles de evidencia de los artículos de soporte y el grado de recomendación de la práctica preventiva realizada.

Palabras clave: Inmunocompromiso, huéspedes especiales, vacuna de neumococo.

\section{INTRODUCCIÓN}

Los trastornos de la inmunocompetencia es un término que a menudo se usa como sinónimo de inmunosupresión, inmunodeficiencia o inmunocompromiso; estas patologías pueden clasificarse como primarias o secundarias y se asocian a situaciones especiales que pueden generar alteración inmunológica en el paciente pediátrico. ${ }^{1}$ La Tabla 1 nos muestra un modelo de su clasificación.

Las inmunodeficiencias primarias generalmente son heredadas e incluyen afecciones definidas por una ausencia inherente o por deficiencia cuantitativa de componentes celulares, humorales o de ambos
For this, both the Guidelines for the development of consensus and the recommendations and criteria of the European AGREE initiated were used. For the formation of the consensus group, mixed and representative teams were integrated by pediatricians, infectious diseases specialists, immunologists, and allergists participated; as well as pediatricians from the private sector. Articles within the period from January 2015 to August 2021 were further selected. Results: A total of 10 relevant points were approached as questions, and a series of recommendations were established based on the critical literature analysis.

Keywords: Immunocompromise, special hosts, pneumococcal vaccination.

que se conocen como responsables de la respuesta inmunológica. Las inmunodeficiencias secundarias incluyen las asociadas con infecciones por patógenos como el virus de la inmunodeficiencia humana $(\mathrm{VIH})$, o neoplasias hematopoyéticas, tratamiento con quimioterapia o radiación y tratamiento con medicamentos quimioterapéuticos.

Por otra parte, ciertas condiciones como la asplenia funcional o quirúrgica, la enfermedad renal crónica o el uso de fármacos inmunosupresores también pueden causar alteraciones en la inmunocompetencia.

Los pacientes inmunocomprometidos son el grupo más afectado y con mayores tasas de mortalidad

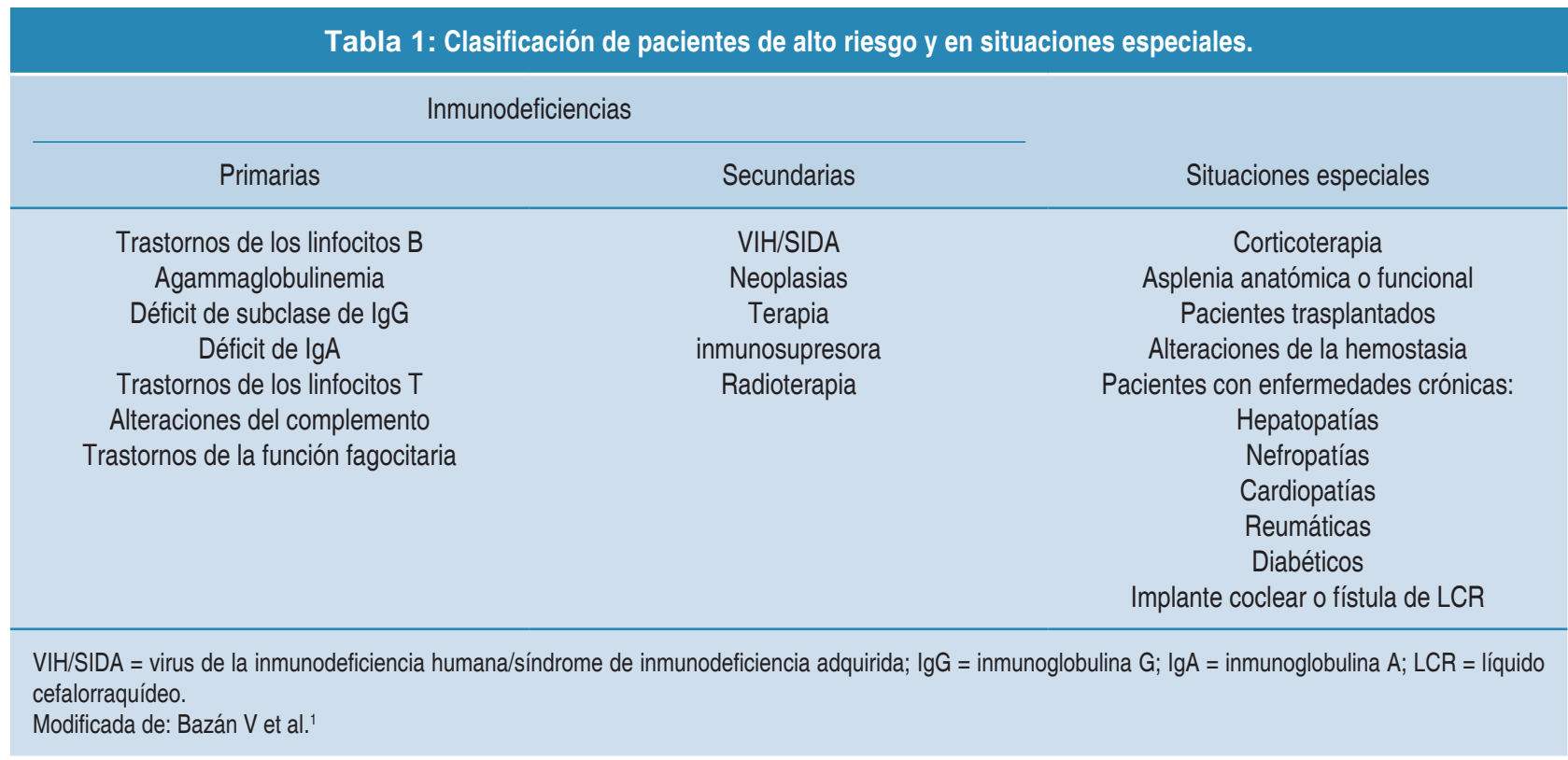


asociada a infecciones invasivas por neumococo, infecciones por meningococo, Haemophilus influenzae tipo b o los virus de la influenza o varicela así como de otros agentes oportunistas.

La vacunación en pacientes con inmunocompromiso, conocidos también como pacientes de alto riesgo, tiene un papel fundamental en la mejoría de la sobrevida y calidad de vida de estos pacientes. Por otra parte, la seguridad y eficacia de estos inmunógenos en pacientes con alteraciones de su respuesta inmunológica va a depender del tipo y grado de inmunosupresión individual, por lo que es de vital importancia conocer cuáles son las vacunas indicadas y cuáles las contraindicadas por su riesgo en estos pacientes. ${ }^{1,2}$

La enfermedad neumocócica sigue siendo una causa común de morbilidad y mortalidad en todo el mundo.

La Organización Mundial de la Salud (OMS) ha informado de un total de 1.6 millones de muertes en todo el mundo causadas por Streptococcus pneumoniae cada año. Más de 250,000 ingresos hospitalarios cada año, situación que se asocia con grave impacto económico en los países de menor desarrollo. Es por esto que, sumado a la indicación de esta inmunización en el paciente pediátrico sano para disminuir la enfermedad invasiva (meningitis, sepsis) y los cuadros de neumonía, otitis media y sinusitis, hoy día hay un creciente interés en recomendar esta inmunización en pacientes pediátricos de alto riesgo de desarrollar la infección grave como en pacientes con asplenia o disfunción esplénica, trastornos del complemento, leucemias, mieloma múltiple, trasplante de células madre, entre otras patologías; no obstante, estos beneficios propuestos y los porcentajes de inmunización global, en especial en estos niños, siguen siendo muy bajos. ${ }^{3}$

El presente consenso surge como parte de los compromisos de la Asociación Mexicana de Infectología Pediátrica (AMIP) en generar y difundir el conocimiento científico entre la comunidad pediátrica mexicana e iberoamericana, conjuntando el esfuerzo de sus agremiados con el de expertos de otras instituciones y asociaciones, con la finalidad de establecer respuestas y recomendaciones a interrogantes y controversias de actualidad en la infectología pediátrica, y este trabajo surge como parte de las recomendaciones generadas por el Consenso de la AMIP sobre el impacto de la vacunación en la disminución de la carga de enfermedad neumocócica en México en 2018 y por la opinión de un panel de expertos publicado en el año 2020.4,5 En ambos documentos se recomienda realizar una revisión y un análisis que permita proponer los esquemas idóneos de vacunación contra Streptococcus pneumoniae en pacientes con inmunodeficiencias primarias o secundarias así como en pacientes con inmunocompromiso asociado con situaciones especiales. Este mismo panel hizo resaltar la necesidad de contemplar comorbilidades que hoy día son muy comunes en México y que rara vez son tomadas en cuenta para esta evaluación, pero que son consideradas como de riesgo incrementado para la salud pública de nuestro país como la obesidad infantil y el asma así como el inmunocompromiso asociado con enfermedades oncológicas, entre otras, y la utilidad potencial que tiene en ellos el uso de la vacuna conjugada de neumococo 13 valente (PCV13), en especial en su esquema de cuatro dosis.

Este consenso analiza y discute el impacto de la vacunación contra neumococo y otras vacunas recomendadas en la actualidad en algunas situaciones del paciente pediátrico de alto riesgo.

\section{MATERIAL Y MÉTODOS}

Se utilizó el modelo de consenso académico que consistió en el análisis y discusión de la bibliografía científica disponible, que en caso necesario se complementó con la opinión de expertos de los tópicos considerados controversiales o cuya evidencia bibliográfica se consideró insuficiente.

Para el desarrollo del presente consenso se siguió la recomendación de la guía Lineamientos para la elaboración de consensos ${ }^{6}$ y las recomendaciones y criterios útiles para la lectura crítica de los artículos seleccionados de la iniciativa europea AGREE (Appraisal of Guidelines for Research \& Evaluation) ${ }^{7,8}$

Para la conformación del grupo se integraron equipos mixtos y representativos en los que participaron pediatras infectólogos miembros de la AMIP, y pediatras con especialidad en inmunología y alergia así como pediatras del sector privado con experiencia probada en vacunación infantil (Figura 1).

En un inicio, los coordinadores de grupo establecieron una estrategia de búsqueda selectiva en la que se obtuvieron los estudios y manuscritos utilizados para esta revisión.

La identificación de los artículos se llevó a cabo usando las palabras clave: Streptococcus pneumoniae, conjugate pneumococcal vaccine y high-risk 
Rev Latin Infect Pediatr. 2021; 34 (4): 162-176
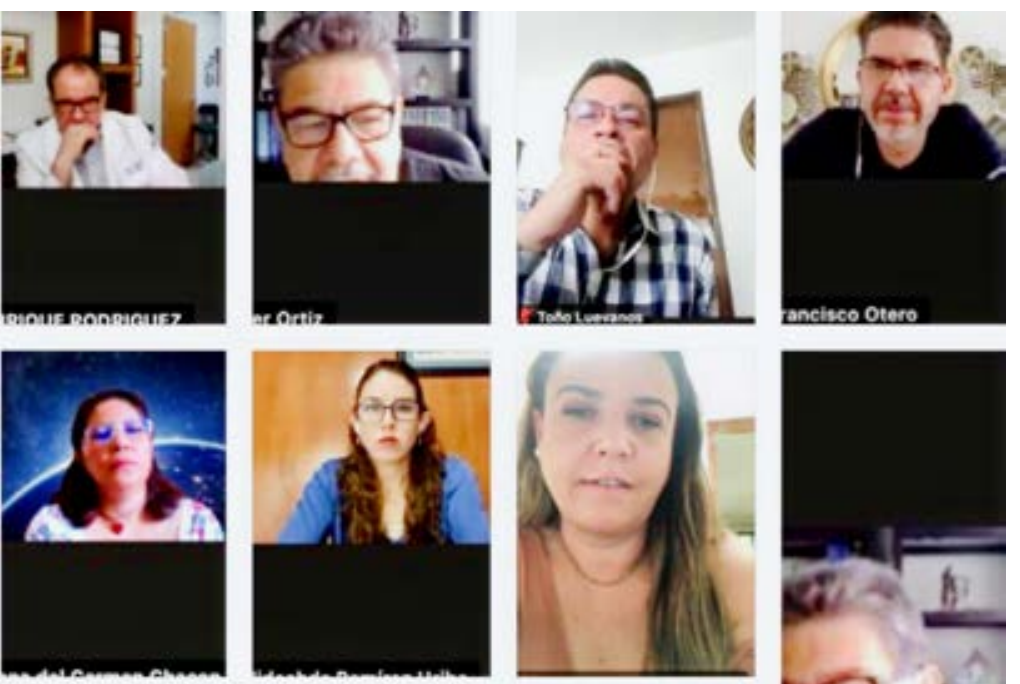

Figura 1:

Grupo colaborativo, Consenso AMIP 2021 virtual. Inmunización con vacuna conjugada de neumococo y otras vacunas recomendadas en pacientes pediátricos de alto riesgo. Ciudad de México, 10 de julio de 2021.
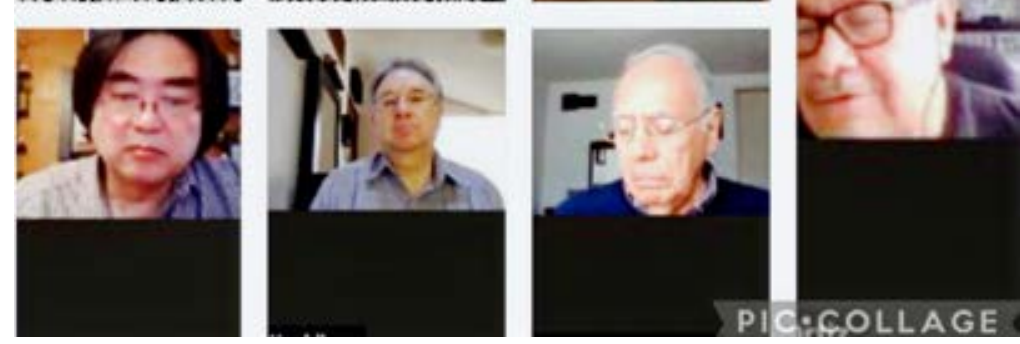

pediatrics, vacunación en huéspedes especiales, vacunación en niños de alto riesgo y otras palabras clave secundarias integradas a la búsqueda, se eligieron los artículos que abarcaran el periodo de enero de 2015 a agosto de 2021. Se seleccionaron artículos publicados en inglés y español en los buscadores PubMed, Google Scholar, Embase y Cochrane así como documentos de la biblioteca digital de la Universidad de Oxford. Durante el desarrollo del consenso se incluyeron artículos de mayor antigüedad por ser estudios originales de importancia o revisiones relacionadas así como consensos y documentos de opinión realizados por expertos en el tema. Se hicieron búsquedas ascendentes usando algunas de las referencias de los artículos seleccionados, en especial en el análisis de estudios de seguimiento a largo plazo.

Una vez identificada la bibliografía a revisar, se organizó una primera reunión de tipo virtual, donde se definió el alcance de los temas a analizar. En esta misma reunión se establecieron las bases para la adopción y adaptación de recomendaciones de otros organismos o sociedades a incluir en las conclusiones del consenso. Se formaron tres mesas de trabajo.
La segunda fase se realizó a distancia entre los grupos participantes y los coordinadores del consenso. Terminada la fase de revisión crítica de la literatura, con los resultados y conclusiones obtenidas por cada grupo, los coordinadores del consenso redactaron el primer manuscrito, que posteriormente fue revisado por la totalidad del grupo.

La validación del manuscrito fue llevada a cabo por dos expertos externos, quienes realizaron una revisión independiente; sus observaciones y recomendaciones formaron parte de la reformulación necesaria y se integraron al documento final. Las respuestas, conclusiones y recomendaciones se presentan con sus niveles de evidencia, grado de recomendación así como su propuesta de aplicabilidad en la práctica clínica.

Las Tablas 2 y 3 nos muestran las escalas de la Canadian Task Force on Preventive Health Care (CTFPHC) elaboradas por la Public Health Agency of Canada (PHAC) para desarrollar guías de práctica clínica que respalden las acciones de salud preventiva y seleccionadas para la evaluación del grado de las recomendaciones emitidas y el nivel de evidencia que las soporta. ${ }^{7,8}$ 
Tabla 2: Grados de recomendación para

intervenciones de prevención (CTFPHC).

Grado de

recomendación Interpretación

A Existe buena evidencia para recomendar la intervención clínica de prevención

B Existe evidencia moderada para recomendar la intervención clínica de prevención

C La evidencia disponible es contradictoria y no permite hacer recomendaciones a favor o en contra de la intervención clínica preventiva; sin embargo, otros factores podrían influir en la decisión

D Existe evidencia moderada para no recomendar la intervención clínica de prevención

E Existe buena evidencia para no recomendar la intervención clínica de prevención

I Existe evidencia insuficiente (cualitativa y cuantitativamente) para hacer una recomendación; sin embargo, otros factores podrían influir en la decisión

En conjunto con el grupo de expertos participantes en la reunión virtual, se seleccionaron 10 aspectos relevantes para la integración crítica y sistemática de las recomendaciones basadas en esta revisión y sesiones de trabajo (Tabla 4).

\section{RESPUESTAS Y RECOMENDACIONES A LAS PREGUNTAS GENERADAS POR EL CONSENSO}

\section{1. ¿Cuáles son las conductas generales sobre vacunación que deben ser consideradas por el vacunador para la aplicación de inmunógenos de prevención primaria o de refuerzos en los pacientes pediátricos con diagnóstico de inmunocompromiso?}

Existe suficiente evidencia para soportar el uso de los esquemas básicos de vacunación así como de algunas inmunizaciones específicas en los pacientes pediátricos de alto riesgo.

Como parte de las conductas generales que todo médico vacunador debe tomar en cuenta al inmunizar a un paciente considerado de riesgo alto de infecciones, este consenso académico recomienda los siguientes lineamientos.

\section{Recomendación del consenso}

1. Salvo indicaciones precisas, se deben evitar las vacunas de microorganismos vivos atenuados en pacientes con estados de inmunoalteración comprobada o de sospecha.

2. Se recomienda administrar vacunas de fracciones o microorganismos inactivados así como el uso de vacunas conjugadas sobre las vacunas de virus vivos o de virus atenuados.

3. Completar esquemas de vacunación o refuerzos antes de entrar a un tratamiento o procedimiento inmunosupresor.

4. Si el paciente recibe hemoderivados tipo inmunoglobulinas, debe esperar al menos tres meses para la aplicación de una vacuna de virus vivos. , $^{2,9,10}$

\section{Nivel de evidencia I, II-2 y III, grado de reco- mendación A}

5. Se debe vacunar a familiares y contactos que convivan de manera habitual con el paciente para la prevención de agentes potencialmente mortales como la influenza, y se recomienda la utilización del esquema de cuatro dosis $(3+1)$ de la vacuna conjugada 13 valente (PCV13) de neumococo en los lactantes que convivan con el paciente afectado (hermanos, primos), con la

\section{Tabla 3: Niveles de evidencia e interpretación de los tipos de estudio para intervenciones de prevención (CTFPHC).}

\begin{tabular}{|c|c|}
\hline $\begin{array}{l}\text { Nivel de } \\
\text { evidencia }\end{array}$ & Interpretación \\
\hline I & $\begin{array}{l}\text { La evidencia existente surge a partir de EC con } \\
\text { asignación aleatoria }\end{array}$ \\
\hline II-1 & $\begin{array}{l}\text { La evidencia existente surge a partir de EC sin } \\
\text { asignación aleatoria }\end{array}$ \\
\hline II-2 & $\begin{array}{l}\text { La evidencia existente surge a partir de estudios } \\
\text { de cohortes y de casos y controles, idealmente } \\
\text { realizados por más de un centro o grupo de } \\
\text { investigación }\end{array}$ \\
\hline II-3 & $\begin{array}{l}\text { La evidencia existente surge a partir de } \\
\text { comparaciones en el tiempo o entre distintos } \\
\text { centros con o sin la intervención; podrían } \\
\text { incluirse resultados provenientes de estudios sin } \\
\text { asignación aleatoria }\end{array}$ \\
\hline III & $\begin{array}{l}\text { La evidencia existente surge a partir de la opinión } \\
\text { de expertos, con base en la experiencia clínica, } \\
\text { estudios descriptivos o informes de comités de } \\
\text { expertos }\end{array}$ \\
\hline
\end{tabular}

EC = ensayo clínico. 
Rev Latin Infect Pediatr. 2021; 34 (4): 162-176

\section{Tabla 4: Preguntas seleccionadas por consenso.}

1. ¿Cuáles son las conductas generales sobre vacunación que deben ser consideradas por el vacunador para la aplicación de inmunógenos de prevención primaria o de refuerzos en los pacientes pediátricos con diagnóstico de inmunocompromiso?

2. ¿Existe mayor riesgo de enfermedad invasiva por Streptococcus pneumoniae en los pacientes con inmunocompromiso?

3. ¿Cuál es la conducta a seguir en relación con la vacunación contra Streptococcus pneumoniae en pacientes pediátricos con diagnóstico de enfermedad oncológica?

4. ¿Los pacientes con síndrome obstructivo bronquial (SOB), bronquitis crónica y/o asma, así como el grupo de fumadores, tienen mayor riesgo de NAC y ENI que las personas sin estas comorbilidades?

5. ¿Qué conducta preventiva se debe seguir en los pacientes con asplenia anatómica o que requieren o requirieron una esplenectomía quirúrgica y cuál es la diferencia de un esquema de vacunación contra neumococo tratándose de esplenectomía electiva versus de urgencia?

6. ¿Los donadores de células progenitoras hematopoyéticas (TCPH) deben recibir alguna inmunización previa al trasplante?

7. ¿Los pacientes postrasplantados de CPH deberán ser vacunados contra COVID-19?

8. ¿Se debe aplicar la vacuna BCG en niños que viven con $\mathrm{VIH}$ ?

9. ¿Cuál es la utilidad conocida de la vacuna de neumococo en pacientes pediátricos que viven con VIH?

10. ¿La obesidad infantil influye en la necesidad de ajustar el esquema de vacunación?

$\mathrm{NAC}=$ neumonía adquirida en la comunidad; $\mathrm{ENI}=$ enfermedad neumocócica invasiva; $\mathrm{CPH}=$ células progenitoras hematopoyéticas; $\mathrm{BCG}=$ bacilo de CalmetteGuérin; VIH = virus de la inmunodeficiencia humana.

intención de erradicar la colonización faríngea de Streptococcus pneumoniae. ${ }^{4}$ Nivel de evidencia III, grado de recomendación B

6. Si se planea la inmunosupresión (no urgente por trasplante de órganos sólidos o inicio de inmunosupresores, terapia para una afección inflamatoria) y si el tiempo lo permite, se recomienda proporcionar todas las vacunas vivas e inactivadas necesarias faltantes al menos cuatro semanas antes de la inmunosupresión.

Las vacunas inactivadas pueden ser administradas al menos dos semanas previas al procedimiento. Para facilitar la inmunización previa al trasplante, se pueden administrar vacunas combinadas como la de sarampión, paperas, rubeola y varicela (SPRV) a candidatos a trasplante de órganos sólidos desde los seis meses de edad, si esto es necesario. ${ }^{2,9,10}$ Nivel de evidencia I, II-2 y III, grado de recomendación A

Si la inmunosupresión es urgente, pero temporal, se recomienda diferir las inmunizaciones hasta que el sistema inmunitario se haya recuperado. Si el riesgo de exposición a una infección específica es alto (ejemplo, temporada de influenza), se pueden administrar vacunas inactivadas, aunque la respuesta puede ser menor.

Las dosis administradas durante la inmunosupresión deben repetirse cuando el sistema inmunitario se haya recuperado.
7. Complicaciones graves han seguido a la inmunización con vacunas vivas atenuadas virales y bacterianas entre personas con inmunocompromiso severo, por lo que la administración de vacunas vivas como SPR, varicela, SPRV, vacuna de varicela/zóster, fiebre amarilla, fiebre tifoidea, Bacilo de Calmette-Guérin (BCG) y viruela debe limitarse sólo a situaciones especiales. ${ }^{2,11}$ Nivel de evidencia III, grado de recomendación A

8. La duración de la respuesta inmunológica puede disminuir para algunas vacunas, por lo que se puede requerir de refuerzos extras (ejemplo: hepatitis B).

\section{2. ¿Existe mayor riesgo de enfermedad invasiva por Streptococcus pneumoniae en los pacientes con inmunocompromiso?}

La frecuencia de la enfermedad neumocócica invasiva (ENI) en la infancia disminuyó drásticamente como resultado de la introducción de la vacuna conjugada neumocócica 7 y 13 valente, situación que debe ser trasladada con prioridad al paciente pediátrico con algún tipo de inmunocompromiso, ya que estos pacientes son el grupo más afectado y con mayores tasas de mortalidad asociada con infecciones neumocócicas invasivas, dentro de estos grupos se pueden mencionar los pacientes con infección por $\mathrm{VIH}$, aquéllos con enfermedad renal crónica, neumópatas crónicos, pacientes con enfermedades cardiacas y quienes tienen 
tratamiento inmunosupresor o para cáncer, ${ }^{1-3}$ ya que estos pacientes son especialmente vulnerables a las enfermedades infecciosas y en particular al Streptococcus pneumoniae, con base en que los títulos de anticuerpos de las enfermedades prevenibles por vacunación disminuyen después del tratamiento intensivo contra el cáncer o enfermedades autoinmunes.

El impacto que ha tenido la vacunación universal en la mortalidad por neumococo se ha evidenciado en diferentes estudios, mostrando que los porcentajes de protección de la vacuna conjugada antineumocócica 13 valente o PCV13 lograron reducir en $67 \%$ los números de casos de enfermedad invasiva. No obstante, se sabe que los serotipos no incluidos en las vacunas PCV 7, 10 y 13 valente son los causantes de la mayor pérdida de años de calidad de vida, los cuales fueron el $22 \mathrm{~F}$ y $33 \mathrm{~F}$ en niños de cinco años y el serotipo 31 en las personas mayores. Estudios realizados en Estados Unidos reportan la reducción de 62.5 y $57.1 \%$ para todos los tipos de presentación de enfermedad neumocócica invasiva con la vacunación antineumocócica 13 valente (PCV13). ${ }^{3,12,13}$ Nivel de evidencia II-2, II-3 y III, grado de recomendación A

Otro de los grupos especialmente susceptibles en niños son los pacientes sometidos a trasplante de células madre hematopoyéticas. En estos pacientes las vacunas podrían ayudar a reducir la morbilidad y la mortalidad debidas a enfermedades prevenibles por vacunación al reducir las tasas y la gravedad de las infecciones y al minimizar las interrupciones del tratamiento contra el cáncer. ${ }^{3,14,15}$ Nivel de evidencia II-2 y III

Sin embargo, las tasas de vacunación contra este agente en pacientes con cáncer y sobrevivientes de cáncer son inaceptablemente bajas y las pautas internacionales se siguen a veces en forma inadecuada. ${ }^{3,4}$

De igual forma, la vacuna conjugada contra neumococo 13 valente es un componente esencial de la protección contra la infección después del trasplante de órganos sólidos, tanto para niños como para adultos, incluso en pacientes con esquemas de vacunación completos o parcialmente concluidos. En general, la vacunación o refuerzos de éstas antes del trasplante da como resultado una mejor respuesta inmunológica, y el periodo antes del trasplante es una oportunidad especial para brindar protección a esta población vulnerable.

\section{Recomendación del consenso}

A pesar de que la respuesta inmunológica a la vacunación contra neumococo en el niño con inmunocompromiso sigue siendo menor que en el niño sano, no es razón para retirar el beneficio de esta inmunización a estos pacientes especiales. ${ }^{16}$

Es por esto que el Comité Asesor sobre Prácticas de Vacunación de los Estados Unidos (ACIP) y la $\mathrm{AMIP}^{2-4}$ recomiendan el uso rutinario de $\mathrm{PCV} 13$ para todos los niños con un estado inmunocomprometido, ya que la eficacia de esta vacuna ha sido demostrada en múltiples estudios.

Con el fundamento anterior, se recomienda aplicar como mínimo una dosis de PCV13 a todo paciente con patología inmunosupresora de fondo, independientemente de cuántas dosis de PCV13 haya recibido antes, o de los medicamentos 0 inmunosupresores que esté tomando, haciendo énfasis en que la respuesta inmunológica es mejor cuando este refuerzo de vacunación se inicia antes de comenzar el manejo inmunosupresor. Grado de recomendación $\mathrm{A}$

\section{3. ¿Cuál es la conducta a seguir en relación con la vacunación contra Streptococcus pneumoniae en pacientes pediátricos con diagnóstico de enfermedad oncológica?}

Tanto la OMS, las Guías de Práctica Clínica de la Red Nacional Integral del Cáncer 2013 y la Sociedad Europea de Oncología Médica ESMO 2013 recomiendan la vacunación antineumococo en pacientes con esplenectomía, en el trasplante de células progenitoras hematopoyéticas (TCPH) y en otros pacientes con cáncer que reciben terapia inmunosupresora. Más recientemente los centros para el Control y la Prevención de Enfermedades (CDC) y el Comité Asesor sobre Prácticas de Inmunización recomiendan el uso secuencial de PCV13 y PPV23 en adultos con esplenectomía, leucemia, linfoma, enfermedad de Hodgkin, malignidad generalizada y mieloma múltiple de PCV13 y PPV23 en pacientes hematológicos y oncológicos en la edad adulta. La población pediátrica con problemas similares presenta características especiales que han retardado la generalización de estas políticas de vacunación contra Streptococcus pneumoniae en diversos grupos con inmunocompromiso, dentro de los cuales se encuentran los pacientes oncológicos. Aunque la respuesta de anticuerpos luego de la 
Rev Latin Infect Pediatr. 2021; 34 (4): 162-176

administración de la vacuna en este grupo podría ser menor en algunos serotipos que la alcanzada en los niños sanos, los títulos de anticuerpos son protectores y la vacuna logra disminuir la incidencia de enfermedad invasora en estos pacientes.

A favor de esta conducta preventiva podemos mencionar que al menos dos estudios de inmunogenicidad de PCV13 que se han realizado en este grupo de pacientes han mostrado una adecuada respuesta inmunogénica.

El primero es de Hung TY y colaboradores, en el cual se midieron los títulos de lgG preadministración y postadministración de PCV13 en 85 niños con cáncer en un grupo etario de uno a 18 años, comparando los niveles entre terapia inmunosupresora activa versus terapia inmunosupresora complementaria. Los resultados de este ensayo clínico fueron que la respuesta serológica fue apropiada en ambos grupos, con diferencias sutiles contra algunos serotipos. Dichos autores concluyeron que la respuesta inmunológica es satisfactoria. ${ }^{17}$ Nivel de evidencia II-2

El segundo estudio es de Jallow S y su grupo, donde se incluyeron niños de 12 a 71 meses con patología inmunosupresora (VIH/SIDA), insuficiencia renal o patología pulmonar crónica y cáncer, y se compararon los niveles geométricos medios de anticuerpos (GMC) con un grupo control de niños sanos tras una o dos dosis de PCV13. Los resultados mostraron que aun con una menor respuesta inmunogénica para algunos serotipos, la respuesta de GMC en niños con inmunodeficiencia probada es óptima, siendo mayor incluso usando un esquema de dos dosis. ${ }^{18}$ Nivel de evidencia I

En México el manual de vacunación de 2017 del Consejo Nacional de Vacunación recomienda que los pacientes oncológicos sin esquema o con esquemas de vacunación incompletos al momento del diagnóstico de cáncer, deben ser vacunados o revacunados con la vacuna conjugada de 13 serotipos (PCV13). ${ }^{11}$ Nivel de evidencia III, grado de recomendación $\mathrm{A}$

Uno de los principales fundamentos para esta política en salud se basa en el estudio mexicano realizado por Soto-Noguerón que incluyó 3,249 aislamientos de S. pneumoniae de 1994 a 2016, en el que se identificaron 175 aislamientos (5.4\%) de pacientes con cáncer, siendo las enfermedades mieloproliferativas las más comunes en los niños, mientras que los tumores sólidos lo fueron en los adultos. El análisis de los serotipos mostró que $60.5 \%$ de los serotipos de Streptococcus pneumoniae obervados con más frecuencia en este estudio están incluidos en la vacuna PCV13. ${ }^{19}$ Nivel de evidencia II-2

Los pacientes con enfermedad hematooncológica muestran mayor riesgo de presentar enfermedad invasiva neumocócica, por lo que deben recibir un esquema secuencial con vacunas de neumococo conjugada 13 valente y vacuna de polisacáridos 23 valente, este esquema difiere del esquema habitual recomendado en los pacientes inmunocompetentes. ${ }^{2,3,9,15}$ Nivel de evidencia II-3 y III, grado de recomendación $\mathbf{A}$

\section{Recomendación del consenso}

Con fundamento en lo anterior, este consenso recomienda aplicar como mínimo una dosis de PCV13 a todo paciente con patología inmunosupresora de fondo, incluyendo los pacientes con cáncer, independientemente de cuántas dosis de PCV13 hayan recibido antes, o de los medicamentos o inmunosupresores que estén tomando, haciendo énfasis en que la respuesta inmunológica es mejor cuando este refuerzo de vacunación se inicia antes de comenzar el manejo inmunosupresor. ${ }^{1-4,18}$ Grado de recomendación A

Los pacientes que hayan recibido un esquema completo de cuatro dosis de PCV 13, deberán recibir la dosis de vacuna de polisacáridos 23 valente a los 24 meses de vida, y un refuerzo de esta misma vacuna a los cinco años de haberse aplicado la primera dosis de vacuna neumocócica 23 valente.

En los pacientes que hayan recibido el esquema $2+1$ de la vacuna PCV13 antes de los dos años, deberán recibir una cuarta dosis al menos ocho semanas después de la última dosis de PCV13 y dos dosis separadas por ocho semanas si sólo recibieron dos o menos vacunas de PCV13. ${ }^{1-3,16}$ Grado de recomendación $\mathrm{A}$

4. ¿Los pacientes con síndrome obstructivo bronquial (SOB), bronquitis crónica y/o asma, así como el grupo de fumadores, tienen mayor riesgo de NAC y ENI que las personas sin estas comorbilidades?

Pacientes con enfermedad respiratoria crónica (EPOC, bronquitis crónica y/o asma así como el grupo de fumadores) tienen mayor riesgo de NAC y ENI que las personas sin estas comorbilidades..$^{20,21}$ Nivel de evidencia I, III, grado de recomendación A 
La revisión de la biblioteca Cochrane del año 2017 concluyó que la vacunación neumocócica proporciona una protección significativa contra la neumonía adquirida en la comunidad, y que reduce la probabilidad de una exacerbación de EPOC, aunque la evidencia no es suficiente para comparar los beneficios de los diferentes tipos de vacunas antineumocócicas, esta revisión sugiere que todas las personas con SOB deben recibir la vacuna antineumocócica para mejorar la protección contra la neumonía adquirida en la comunidad y para reducir la posibilidad de una exacerbación aguda. ${ }^{20}$ Nivel de evidencia I, grado de recomendación A

Las infecciones endobronquiales crónicas y la infiltración neutrofílica de las vías aéreas inferiores que acompaña a la infección por Streptococcus pneumoniae son otro ejemplo de complicaciones graves. La neumonía recurrente es otro factor de riesgo de la enfermedad pulmonar supurativa crónica (CSLD) y la bronquiectasia, esta última asociada a muerte prematura en niños y adultos. Aunque el Haemophilus influenzae no tipificable es el principal patógeno en estas patologías, los neumococos se aíslan comúnmente de las vías aéreas inferiores de los niños con estos diagnósticos. ${ }^{21}$

Snijders y colaboradores en un estudio retrospectivo evaluaron la influencia de la introducción de la vacuna PCV7 en Italia en los cultivos positivos obtenidos por lavado broncoalveolar (BAL) en niños con enfermedad pulmonar crónica. De un total de 538 cultivos obtenidos por BAL de 515 niños, se detectó un agente infeccioso en $33 \%$ de los casos, de los cuales $51 \%$ fue Haemophilus influenzae, $21 \%$ Streptococcus pneumoniae y $3 \%$ Moraxella catarrhalis. Se observó que después del periodo de vacunación con PCV7, la presencia de Streptococcus pneumoniae disminuyó su recuperación a sólo $12.9 \%$, concluyendo que la vacuna PCV7 en niños con enfermedad pulmonar crónica parece haber cambiado la presencia de Streptococcus pneumoniae en cultivos obtenidos por BAL, aunque esta reducción no fue estadísticamente significativa. ${ }^{22}$ Nivel de evidencia II-3

Otro estudio realizado por Spositoa y colaboradores utilizando técnicas de biología molecular mostró que Streptococcus pneumoniae coloniza a nivel oral alrededor de $20 \%$ de los pacientes con fibrosis quística, concluyendo que el estado del portador de $S$. pneumoniae en niños en edad escolar y adolescentes con $F Q$ es más frecuente de lo que se pensaba anteriormente, y que la vacunación con
PCV13 administrada en el primer año de vida aun con el esquema $3+1$ en estos pacientes no reduce el riesgo de recolonización en la infancia y adolescencia, lo que sugiere la necesidad de establecer acciones preventivas como la utilización de refuerzos o profilaxis antibiótica. ${ }^{22,23}$ Nivel de evidencia II-2, II-3

En el caso de los pacientes con asma, la revisión de Zaidi propone que este riesgo aumentado es impulsado por una mayor prevalencia del portador de $S$. pneumoniae con una respuesta inmunológica inadecuada por la exposición a la bacteria, o una respuesta subóptima a la vacunación. A esto se suma el riesgo de los corticosteroides inhalados que aumentan la posibilidad de neumonía. ${ }^{24}$ Nivel de evidencia III

El estudio prospectivo de Copenhague sobre el asma en la infancia es un estudio de cohorte desde el nacimiento en un solo centro que analizó a hijos de madres asmáticas, en el que se reclutaron desde el nacimiento 411 neonatos hasta los dos años de vida y mostró que los lactantes colonizados con $S$. pneumoniae tuvieron mayor riesgo de un primer episodio de bronquiolitis con desarrollo de sibilancias 0 asma persistente y hospitalización. ${ }^{25}$ Nivel de evidencia II-2

El estudio de Eisenlohr y colaboradores sugiere que los refuerzos de vacunación con vacuna conjugada de neumococo son útiles en la prevención de niños con exacerbaciones por asma, en especial los asmáticos que presentan baja respuesta de anticuerpos a los serotipos de vacuna de neumococo, ya que disminuyen las exacerbaciones asmáticas que requieren corticoesteroides ${ }^{26}$ Nivel de evidencia II-2

Recientemente una revisión sistemática y un metaanálisis realizados por Murray demuestran que la vacuna de influenza previene las hospitalizaciones y visitas a urgencias médicas por crisis asmáticas en 59 a $78 \%$, por lo que la vacunación anual contra influenza debe recomendarse en todos los pacientes asmáticos. ${ }^{27}$ Nivel de evidencia II-2

\section{Recomendación del consenso}

Con base en éstas y otras evidencias, el ACIP así como la AMIP ${ }^{4,28,29}$ insisten en la utilización de cuatro dosis de vacuna PCV13 en los primeros 18 meses de vida con los ajustes necesarios, de acuerdo con el cumplimiento o no de los esquemas en pacientes conocidos con enfermedades respiratorias crónicas.

\section{Grado de recomendación A}


Rev Latin Infect Pediatr. 2021; 34 (4): 162-176

Con base en estas evidencias, este consenso se apega a las recomendaciones de la ACIP para situaciones especiales como enfermedad pulmonar crónica (incluyendo asma tratada con dosis altas de corticosteroides orales) ${ }^{2}$ que aconsejan aplicar en niños menores de cinco años ante una serie de $2+1$ una dosis de refuerzo de PCV13 (al menos ocho semanas después de la última dosis previa de PCV13).

Y si la serie de vacunación es menor de tres dosis de PCV13, se recomienda la aplicación de dos dosis de PCV13 (la primera ocho semanas después de la dosis más reciente y la segunda administrada con ocho semanas de diferencia de esta dosis).

Si el paciente es mayor de dos años y no tiene antecedentes de aplicación de vacuna de polisacáridos 23 valente, se recomienda una dosis de PPSV23 (al menos ocho semanas después de la última dosis de PCV13). Grado de recomendación A

5. ¿Qué conducta preventiva se debe seguir en los pacientes con asplenia anatómica o que requieren o requirieron una esplenectomía quirúrgica y cuál es la diferencia de un esquema de vacunación contra neumococo tratándose de esplenectomía electiva versus de urgencia?

Tanto la asplenia congénita como la asplenia funcional, incluyendo la enfermedad de células falciformes, y la asplenia quirúrgica predisponen al paciente pediátrico que las padece a mayor número de infecciones, en especial por microorganismos capsulados con Streptococcus pneumoniae, meningococo y Haemophilus influenzae tipo B, por lo que una recomendación internacional es vacunar contra estos patógenos a los pacientes que se identifiquen con este padecimiento o que requieran de una extirpación quirúrgica del bazo.

Los niños menores de cinco años con asplenia anatómica congénita deben recibir el esquema convencional de cuatro dosis $(3+1)$ de vacuna conjugada 13 valente (PCV13) contra Streptococcus pneumoniae, iniciando a la edad mínima de seis semanas para la vacuna antineumocócica conjugada.

Se recomiendan cuatro dosis de PCV13 para todos los niños menores de cinco años, con un esquema primario de dos, cuatro y seis meses; la cuarta dosis al año de vida y a los dos años se deberá iniciar la vacuna antineumocócica de polisacáridos 23 valente (PPSV23). Ésta deberá administrase por lo menos cuatro semanas después de la última dosis de PCV13, en especial a los niños que fueron vacunados con su cuarta dosis cercanos a cumplir los dos años y revacunarse con PPSV23 cinco años después. ${ }^{1,2,28}$ Nivel de evidencia II-3, grado de recomendación $\mathrm{A}$

Se recomienda administrar una serie primaria de dos dosis de MenACWY-CRM o MenACWY-D a personas de dos años o mayores con asplenia o deficiencia del complemento. Después de la serie primaria de vacuna, se recomienda un intervalo de tres años a la siguiente dosis para las personas que recibieron la dosis antes de los siete años y un intervalo de cinco años para las personas que recibieron la dosis después de los siete años o más. ${ }^{11}$

Aunque MPSV4 es la única vacuna contra el meningococo autorizada para personas mayores de 55 años, los adultos mayores de 56 años con asplenia o deficiencia del complemento se pueden vacunar con MenACWY-CRM o MenACWY-D en lugar de MPSV4. ${ }^{30}$ Nivel de evidencia III

En el grupo de niños menores de cinco años se deben administrar las tres dosis correspondientes a la vacuna pentavalente acelular así como la hexavalente y la vacuna de Haemophilus influenzae b; en caso necesario, el intervalo puede acortarse a seis semanas en la serie primaria y se requiere un refuerzo a los 18 meses de edad. Los niños con asplenia o que serán sometidos a extirpación quirúrgica del bazo y no tengan el esquema completo, deberán recibir al menos una dosis de vacuna contra Haemophilus influenzae b previo al evento quirúrgico. ${ }^{11,29}$ Nivel de evidencia III

En los casos de asplenia quirúrgica electiva, las vacunas contra PCV13, contra meningococo y Haemophilus influenzae b deberán aplicarse idealmente al menos dos semanas previas a la intervención planeada; sin embargo, si no se dispone de este tiempo se deberá aplicar lo antes posible al evento quirúrgico. ${ }^{31}$ Nivel de evidencia II-1

Para la vacuna MenACWY-D se recomienda que al menos debe haber un tiempo de cuatro a 12 semanas entre la última aplicación de neumococo $13 \mathrm{~V}$ y esta vacuna, este intervalo no aplica para las vacunas MenACWY-CRM y MenACWY-TT.

Si las vacunas no se administran antes de la cirugía, en especial en los casos de trauma abdominal con extirpación de urgencia del bazo, deberán aplicarse posterior a la vacuna PCV13 en los primeros siete a 10 días después del evento quirúrgico. Es altamente recomendable hacerlo antes del alta quirúrgica realizando un refuerzo a las ocho 
semanas, desafortunadamente con frecuencia se olvida la práctica de este refuerzo.

Por último, si el paciente ha superado estos tiempos postquirúrgicos para la aplicación ideal y es detectado en la consulta clínica como paciente de riesgo por ausencia de bazo, se deberá aplicar las dosis ya mencionadas, ya que el beneficio observado siempre será mayor contra el riesgo latente de enfermedades invasivas por estos microorganismos. ${ }^{32}$ Nivel de evidencia II-3

\section{Recomendación del consenso}

Las personas con asplenia funcional o anatómica (incluida la enfermedad de células falciformes) y deficiencia persistente de componentes del complemento (incluidas las personas que toman eculizumab) tienen mayor riesgo de enfermedad por neumococo y meningococo, por lo que deben recibir un esquema secuencial con cuatro dosis de la vacuna PCV13 y posteriormente la vacuna PPS23V, además de las vacunas contra meningococo MenACWY y MenB. Para los niños de dos a 23 meses de edad se debe administrar una serie de vacuna conjugada contra el meningococo apropiada para la edad. Se recomienda administrar MenACWY-D, que es la vacuna disponible en México, a un niño con asplenia después de los dos años y al menos cuatro semanas después de completar todas las dosis de PCV13. Grado de recomendación A

\section{6. ¿Los donadores de células progenitoras hematopoyéticas (TCPH) deben recibir alguna inmunización previa al trasplante?}

El trasplante de células hematopoyéticas consiste en la transferencia de estas células de un individuo a otro (trasplante alogénico) o el retorno de células colectadas de un individuo a sí mismo (trasplante autólogo), posterior a la manipulación de las células y del receptor. Hoy día aún existen controversias sobre los tiempos y cuáles vacunas deben ser recomendadas tanto en los donadores como en los receptores.

Los candidatos a trasplante deberán recibir las vacunas indicadas para personas sanas basadas en edad, historia de inmunizaciones, factores individuales de riesgo e historia de exposición, según los esquemas recomendados, y deberán contar con un intervalo de aplicación previo al acondicionamiento quimioterapéutico igual o mayor de cuatro semanas para vacunas vivas atenuadas e igual o mayor de dos semanas para vacunas inactivadas. ${ }^{33,34}$ Nivel de evidencia III, grado de recomendación A

Aun cuando existen ciertas bases teóricas en cuanto a que la vacunación del donante de precursores hematopoyéticos en determinados casos constituya una estrategia que pueda mejorar la respuesta a vacunas del receptor, hay escasa evidencia con estudios controlados que puedan soportar esta recomendación como una práctica efectiva, por lo que este consenso no recomienda la vacunación del donador previo al trasplante, ya que además parece ser una práctica de poco beneficio clínico. ${ }^{34}$ Grado de recomendación $\mathrm{D}$

Sin embargo, bajo este marco de ausencia de evidencia teórica se deja al criterio médico la decisión de la aplicación de vacunas al donante; sin embargo, se recomienda evitar la aplicación de vacunas de virus vivos atenuados como sarampión, paperas y rubeola (SPR) así como sarampión, paperas, rubeola y varicela (SPRV) y las vacunas individuales de varicela y zóster en las cuatro semanas previas a la obtención o extracción de los precursores hematopoyéticos. ${ }^{33-35}$ Grado de recomendación $\mathbf{C}$

\section{7. ¿Los pacientes postrasplantados de $\mathrm{CPH}$ deberán ser vacunados contra COVID-19?}

La pandemia del coronavirus 2 (SARS-CoV-2) continúa causando un exceso de morbilidad y mortalidad en el mundo. Los receptores de células $T$ de trasplante de células hematopoyéticas (HCT) y receptor de antígeno quimérico (CAR) tienen mayor riesgo de sufrir complicaciones graves por este virus, por lo que ante este riesgo la administración de las vacunas de COVID-19 a los tres a seis meses después del trasplante, más aún si la tasa de transmisibilidad en el entorno es alta, debe ser una práctica recomendada incluso a pesar de que la eficacia observada con estas vacunas podría ser baja. Las vacunas de ARNm SARS-CoV-2 pueden ofrecerse de manera temprana en receptores de $\mathrm{TCPH}$ o terapia CAR-T (receptor de antígeno quimérico de células T) para prevenir infecciones y enfermedad grave; a los receptores de TCPH y terapia celular CAR-T programados para recibir terapia citotóxica o depleción de células $B$ se les podrá ofrecer la vacuna de COVID-19 previa a la intervención y dejar por lo menos dos semanas después de la segunda dosis dentro de lo posible para facilitar la formación de 
Rev Latin Infect Pediatr. 2021; 34 (4): 162-176

células T de memoria previa al inicio de las terapias citotóxicas o de depleción de células $B$. Pese a la escasa experiencia que aún hay en este escenario, estas vacunas parecen además mostrar un perfil adecuado de seguridad e inmunogenicidad en estos pacientes. ${ }^{36,37}$ Nivel de evidencia III, grado de recomendación B

\section{Recomendaciones del consenso}

Este consenso considera que debido al alto riesgo de padecer enfermedad grave o muerte por COVID-19 en los pacientes receptores de TCPH, las vacunas de ARNm SARS-CoV-2 contra COVID, aun con la escasa información generada, deben recomendarse en este grupo especial de pacientes. Nivel de evidencia II-1 y III, grado de recomendación B

De igual forma, ante el riesgo elevado de padecer enfermedad invasiva por neumococo en los pacientes receptores de TCPH, este consenso recomienda aplicar la vacuna de neumococo conjugada 13 valente PCV13 entre los primeros tres a seis meses del TCPH. Si el paciente está estable sin enfermedad de injerto contra hospedero severa $(\mathrm{EICH})$, se recomienda la aplicación de al menos tres dosis de PVC13 y una adicional al año de PPSV 23V. En caso de inmunocompromiso o $\mathrm{EICH}$ severos, se recomienda aplicar cuatro dosis de PCV13 en el esquema $3+1$. Nivel de evidencia III, grado de recomendación $B$

Vacunas en controversia en niños que viven con VIH

8. ¿Se debe aplicar la vacuna BCG en niños que viven con VIH?

Las recomendaciones de inmunizaciones en el niño con VIH han sufrido modificaciones a través del tiempo, en especial con el surgimiento de nuevos y mejores tratamientos en los grupos pediátricos que han logrado mejorar no sólo su sobrevida, sino además su calidad de vida y mantener niveles de inmunidad cada vez mayores.

La OMS recomienda aplicar la vacuna BCG al nacer en todos los niños asintomáticos expuestos al VIH en países con alta prevalencia de tuberculosis. No obstante, de acuerdo con diversas revisiones y para evitar complicaciones se sugiere en primer lugar descartar si hubo o no transmisión de VIH por vía perinatal antes de su aplicación (aproximadamente a las seis semanas de vida). Si se comprueba la transmisión perinatal, se sugiere diferir la aplicación de la vacuna BCG e iniciar la terapia antirretroviral para evitar el síndrome de reconstitución inmune que podría ser inducido por la vacuna BCG. ${ }^{38-41}$ Nivel de evidencia I, II-2 y III, grado de recomendación $A$

\section{9. ¿Cuál es la utilidad conocida de la vacuna de neumococo en pacientes pediátricos que viven con VIH?}

Neumococo es el principal agente capsulado causante de enfermedades invasivas en los niños que viven con VIH ante su deficiencia opsónica mediada por IgG específica, muy relacionada con bajo nivel de CD4 y carga viral elevada. Asimismo, tienen mayor posibilidad de padecer enfermedades no invasivas.

La inmunogenicidad de las vacunas neumocócicas conjugadas, aunque menor que en los pacientes sanos, es aceptable y entre $76-96 \%$ de los niños con $\mathrm{VIH}$, ya que alcanzan concentraciones protectoras. ${ }^{3,41-44}$ Nivel de evidencia II-2, II-3, III, grado de recomendación $\mathbf{A}$

\section{Recomendaciones del consenso}

Con base en el beneficio de una adecuada inmunogenicidad por las vacunas conjugadas de 13 serotipos de neumococo en los pacientes pediátricos que viven con $\mathrm{VIH}$, la indicación de esta vacuna debe ser obligatoria; no obstante, por observarse menores porcentajes de respuesta con el esquema $2+1$, se recomienda utilizar el esquema de cuatro dosis en estos pacientes a los de dos, cuatro, seis y 15 meses. Grado de recomendación A

\section{0. ¿La obesidad infantil influye en la necesidad de ajustar el esquema de vacunación?}

La creciente prevalencia de la obesidad infantil se ha convertido en un grave problema de salud durante las últimas décadas.

La obesidad infantil generalmente se asocia a un proceso de inflamación crónica persistente, siendo considerada hoy día como una epidemia mundial. Se conoce que la obesidad causa una desregulación en todo el sistema inmunológico, lo que afecta el equilibrio y los niveles de citocinas, adipocinas y células inmunitarias innatas y adaptativas. Esta 
función inmunológica en los niños altera el estado de activación inicial de las células inmunitarias y afecta la capacidad del huésped para combatir patógenos y tumores malignos, además de presentar una respuesta disminuida al reto inmunogénico de la vacunación. ${ }^{45}$ Nivel de evidencia III

Un escrutinio sistemático de revisiones realizadas en 2017 por Roya Kelishadi aborda los hallazgos de los artículos de revisión sobre la relación que existe entre la obesidad infantil y materna y el sistema inmunológico de los niños. Una metarrevisión de 26 artículos realizada en 2017 por Roya Kelishadi aborda los hallazgos sobre la relación que existe entre la obesidad infantil y materna, y el sistema inmunológico de los niños. Los resultados observados sugieren que la obesidad infantil está asociada con cambios extensos en los niveles séricos de citocinas y proteínas inflamatorias y antiinflamatorias así como en la cantidad de células inmunes y su comportamiento, por lo que esto podría causar o exacerbar enfermedades como asma, alergia, dermatitis atópica (EA) y síndrome de apnea obstructiva del sueño. Además, la obesidad infantil puede reducir la capacidad de respuesta del sistema inmunológico a las vacunas y microorganismos. ${ }^{46}$

\section{Nivel de evidencia II-3}

No obstante, los datos sobre el efecto de la obesidad en la inmunogenicidad y la eficacia de la vacuna aún son limitados. Sin embargo, éstos sugieren que la obesidad es un factor que aumenta la probabilidad de una respuesta inmunológica deficiente inducida por las vacunas. ${ }^{47}$

La obesidad se ha asociado con respuestas atenuadas a las vacunas y con mayor riesgo de contraer neumonía neumocócica, pero ningún estudio, hasta donde sabemos, ha evaluado el impacto de la obesidad y la genética en la eficacia de la vacuna antineumocócica conjugada (PCV13).

En el estudio de Sebastian M y colaboradores se evaluó la relación de la obesidad (análisis primario) y el genotipo estimulador de genes de interferón (STING1) (análisis secundario) con la eficacia de la vacuna polisacárida 23 valente PPSV23 utilizando muestras de suero para evaluar los anticuerpos específicos de PPSV23. Los genotipos de STING1 se identificaron mediante PCR en ADN extraído de muestras de sangre periférica. ${ }^{48}$

Se observó que los participantes obesos tuvieron mayor cambio en las respuestas específicas de la vacuna en comparación con los participantes no obesos $(p<0.0001)$. Este grupo de estudio concluye que sus observaciones demuestran una asociación positiva entre la obesidad y la disminución de la eficacia de PPSV23, específicamente en participantes con el genotipo WT STING1. Nivel de evidencia II-1

\section{Recomendaciones del consenso}

Este conceso concluye que aunque la evidencia encontrada apunta a que la obesidad puede ser un factor en la disminución de la respuesta inmunológica a las vacunas de neumococo, esta evidencia aún es escasa, por lo que se considera esperar a reunir un mayor número de estudios que permitan recomendar alguna modificación sobre los esquemas de vacunación en el niño sano. Grado de recomendación $B$

\section{REFERENCIAS}

1. Bazán V, Castellano V, Lamy P, Nolte F, Pacchiotti A, Aversa $L$ et al. Guía de vacunación en huéspedes especiales. Rev Hosp Niños. 2018; 60 (268): 96-110.

2. Altered Immunocompetence. General Best Practice Guidelines for Immunization: Best Practices Guidance of the Advisory Committee on Immunization Practices (ACIP). Available in: https://www.cdc.gov/vaccines/hcp/acip-recs/ general-recs/immunocompetence.html

3. Froneman C, Kelleher P, José RJ. Pneumococcal vaccination in immunocompromised hosts: an update. Vaccines (Basel). 2021; 9 (6): 536. doi: 10.3390/vaccines9060536.

4. Ortiz-Ibarra FJ, Luévanos-Velázquez A, González SN, Reyna-Figueroa J, Chacón CE, Echaniz AG et al. Consenso de la Asociación Mexicana de Infectología Pediátrica (AMIP) sobre el impacto de la vacunación en la disminución de la carga de enfermedad neumocócica en México, 2018. Rev Latin Infect Pediatr. 2018; 31: 62-75.

5. Xochihua-Díaz L, Ortiz-lbarra FJ, González Saldaña N. Streptococcus pneumoniae: recomendaciones de un panel de expertos, AMIP 2020. Rev Latin Infect Pediatr. 2021; 34 (1): 17-19.

6. Ferrario C, Califano G, Durán P, Maccarone M, Miceli I, Manterola A et al. Lineamientos para la elaboración de consensos. Arch Argent Pediatr. 2012; 110 (2): 163-167. doi: org/10.5546/aap.2012.163.

7. The AGREE Collaboration. Appraisal of Guidelines for Research \& Evaluation (AGREE) Instrument. Available in: https://www. agreetrust.org/resource-centre/the-originalagree-instrument/

8. Manterola C, Asenio-Lobos C, Otze T. Jerarquización de la evidencia. Niveles de evidencia y grados de recomendación de uso actual. Rev Chilena Infectol. 2014; 31: 705-718.

9. Dulek DE, de St Maurice A, Halasa NB. Vaccines in pediatric transplant recipients-Past, present, and future. Pediatr Transplant. 2018; 22 (7): e13282. doi: 10.1111/petr.13282.

10. Moore DL. Immunization of the immunocompromised child: key principles. Paediatr Child Health. 2018; 23 (3): 203-205. doi: $10.1093 / p c h / p x x 180$.

11. Manual de Vacunación 2017 Centro Nacional para la Salud de la Infancia y la Adolescencia. Disponible en: http://bit. ly/2n1vF8S 
Rev Latin Infect Pediatr. 2021; 34 (4): 162-176

12. Hsu HE, Shutt KA, Moore MR et al. Effect of pneumococcal conjugate vaccine on pneumococcal meningitis. $\mathrm{N}$ Engl $\mathrm{J}$ Med. 2009; 360: 244-256.

13. Grijalva CG, Nuorti JP, Arbogast PG, Martin SW, Edwards $\mathrm{KM}$, Griffin MR. Decline in pneumonia admissions after routine childhood immunization with pneumococcal conjugate vaccine in the USA: a time- series analysis. Lancet. 2007; 369: 1179-1186.

14. Danziger-Isakov L, Kotton CN. Vaccination in organ transplant patients. In: Safdar A (eds). Principles and practice of transplant infectious diseases. New York, NY: Springer; 2019.

15. Weber T, Ljungman P. Stringent vaccination of cancer patients: is it that important? Ann Oncol. 2018; 29 (6): 1348-1349.

16. Chilson E, Scott DA, Schmoele-Thoma B, Watson W, Moran MM, Isturiz R. Immunogenicity and safety of the 13-valent pneumococcal conjugate vaccine in patients with immunocompromising conditions: a review of available evidence. Hum Vaccin Immunother. 2020; 16 (11): 27582772.

17. Hung TY, Kotecha RS, Blyth CC, Steed SK, Thornton RB, Ryan AL et al. Immunogenicity and safety of single dose, 13-valent pneumococcal conjugate vaccine in pediatric and adolescent oncology patients. Cancer. 2017; 123: 42154221.

18. Jallow S, Madhi SA, Madimabe R, Sipambo N, Violari A, Kala $U$ et al. Immunogenicity of 13-valent pneumococcal conjugate vaccine among children with underlying conditions. Vaccine. 2017; 35 (34): 4321-4329.

19. Soto-Noguerón A, Carnalla-Barajas NM, Cornejo-Juárez $P$, Volkow-Fernández $\mathrm{P}$, Velázquez-Meza ME, Echániz-Aviles G. Streptococcus pneumoniae: distribution of serotypes and antimicrobial susceptibility in patients with cancer. Salud Pública Mex. 2018; 60 (1): 21-28.

20. Torres A, Blasi F, Dartois N, Akova M. Which individuals are at increased risk of pneumococcal disease and why? Impact of COPD, asthma, smoking, diabetes, and/or chronic heart disease on community-acquired pneumonia and invasive pneumococcal disease. Thorax. 2015; 70: 984-989.

21. Walters JA, Tang JN, Poole P, Wood-Baker R. Pneumococcal vaccines for preventing pneumonia in chronic obstructive pulmonary disease. Cochrane Database Syst Rev. 2017; 1 (1): CD001390. doi: 10.1002/14651858. CD001390.pub4.

22. Snijders D, Masiero S, Da Dalt L, Barbato A. Did the introduction of pneumococcal vaccination in Italy change the BAL positive cultures in children with chronic lung diseases? Eur Respir J. 2016; 48 (suppl 60): PA3145. doi: 10.1183/13993003.congress-2016.PA3145.

23. Esposito S, Colombo C, Tosco A, Montemitro E, Volpi S, Ruggiero $L$ et al. Streptococcus pneumoniae oropharyngeal colonization in children and adolescents with cystic fibrosis. J Cyst Fibros. 2016; 15 (3): 366-371.

24. Zaidi SR, Blakey JD. Why are people with asthma susceptible to pneumonia? A review of factors related to upper airway bacteria. Respirology. 2019; 24: 423-430.

25. Bisgaard $\mathrm{H}$. The copenhagen prospective study on asthma in childhood (COPSAC): design, rationale, and baseline data from a longitudinal birth cohort study. Ann Allergy Asthma Immunol. 2004; 93 (4): 381-389. doi: 10.1016/ S1081-1206(10)61398-1.

26. Eisenlohr CP, Chartrand EM, Barzaga MR, Lanz MJ. Impact of pneumococcal vaccine response on asthma exacerbation frequency in young children. Immun Inflamm Dis. 2020; 8 (4): 493-496. doi: 10.1002/iid3.331.

27. Murray CS, Jackson DJ, Teague WG. Prevention and outpatient treatment of asthma exacerbations in children. J Allergy Clin Immunol Pract. 2021; 9 (7): 2567-2576. doi: 10.1016/j.jaip.2021.03.035. Erratum in: J Allergy Clin Immunol Pract. 2021; 9 (10): 3851.

28. CDC. Recommended Child and Adolescent Immunization Schedule for ages 18 years or younger, United States, 2021. Available in: https://www.cdc.gov/vaccines/schedules/hcp/ imz/child-adolescent.html

29. Luévanos Velázquez A, Mascareñas de los Santos A, González Saldaña N, Ortiz lbarra FJ, Romero Feregrino R, Vázquez Narváez JA et al. Esquema de vacunación recomendado para niños de cero a 12 años en México (2018-2019) Asociación Mexicana de Infectología Pediátrica, AC. Rev Latin Infect Pediatr. 2018; 31 (3): 939694.

30. Gutiérrez-Robledo LM, Caro-López E, Guerrero-Almeida ML, Dehesa-Violante M, Rodríguez-Noriega E, García-Lara JM et al. Resultados del Primer Consenso Mexicano de Vacunación en el Adulto. Gac Med Mex. 2017; 153: 190-204.

31. Alvarado AR, Udobi K, Berry S, Assmann J, McDonald T, Winfield RD. An opportunity for improvement in trauma care: 8-week booster vaccination adherence among patients after trauma splenectomy. Surgery. 2018; 163 (2): 415-418.

32. Luu S, Dendle C, Jones P, Ojaimi S, Woolley IJ. Impact of a spleen registry on optimal post-splenectomy vaccination and care. Hum Vaccin Immunother. 2018; 14 (12): 2894-2899.

33. Rubin LG, Levin MJ, Ljungman $P$ et al. 2013 IDSA clinical practice guideline for vaccination of the immunocompromised host. Clin Infect Dis. 2014; 58: 309-318.

34. Espinoza-Mora MR, Lazo-Paez G, León-Bratti MP, Schauer C. Vacunación de pacientes adultos receptores de trasplante de células madre hematopoyéticas. Perspectiva de Costa Rica. Rev Chilena Infectol. 2016; 33 (6): 635-649.

35. Tomblyn M, Chiller T, Einsele $\mathrm{H}$ et al. Guidelines for preventing infectious complications among hematopoietic cell transplantation recipients: a global perspective. Biol Blood Marrow Transplant. 2009; 15: 1143-1238.

36. American Society of Hematology. ASH-ASTCT COVID-19 Vaccination for HCT and CAR T Cell Recipients: Frequently Asked Questions. Available in: https://www.hematology.org/ covid-19/ash-astct-covid-19-vaccination-for-hct-and-car-tcell-recipients

37. Le Bourgeois A, Coste-Burel M, Guillaume T et al. Safety and antibody response after 1 and 2 doses of BNT162b2 mRNA vaccine in recipients of allogeneic hematopoietic stem cell transplant. JAMA Netw Open. 2021; 4 (9): e2126344. doi: 10.1001/jamanetworkopen.2021.26344.

38. Nuttall JJ, Eley BS. BCG Vaccination in HIV-infected children. Tuberc Res Treat. 2011; 2011: 712736. doi: $10.1155 / 2011 / 712736$

39. Miranda-Choque Edwin, Candela-Herrera Jorge, R. Segura Eddy, Farfán-Ramos Sonia, Barriga Aldo. Síndrome de reconstitución inmune por BCG en niños tratados por VIH. Rev. perú. med. exp. salud publica [Internet]. 2012 Oct [citado 2021 Dic 01] ; 29( 4 ): 498-502. Disponible en: http://www.scielo.org.pe/scielo.php?script=sci_ arttext\&pid=S1726-46342012000400012\&lng=es

40. Kilapandal Venkatraman SM, Sivanandham R, Pandrea I, Apetrei C. BCG Vaccination and mother-to-infant transmission of HIV. J Infect Dis. 2020; 222 (1): 1-3. 
41. Comité Asesor de Vacunas (CAV-AEP). Manual de vacunas en línea de la AEP [Internet]. Madrid: AEP; 2021. Disponible en: http://vacunasaep.org/documentos/manual/manual-devacunas

42. Wysocki J, Brzostek J, Konior R, Panzer FG, Francois NA, Ravula SM et al. Antibody persistence and immunologic memory in children vaccinated with 4 doses of pneumococcal conjugate vaccines: results from 2 long-term follow-up studies. Hum Vaccin Immunother. 2017; 13 (3): 661-675.

43. Wahl B, O'Brien KL, Greenbaum A, Majunder A, Liu L, Chu Y et al. Burden of Streptococcus pneumoniae and Haemophilus influenzae type $b$ disease in children in the era of conjugate vaccines: global, regional, and national estimates for 2000 15. Lancet Glob Health. 2018; 6: e744-757.

44. Adetokunboh OO, Ndwandwe D, Awotiwon A, Uthman OA, Wiysonge CS. Vaccination among HIV-infected, HIV-exposed uninfected and HIV-uninfected children: a systematic review and meta-analysis of evidence related to vaccine efficacy and effectiveness. Hum Vaccin Immunother. 2019; 15 (11): 2578-2589.

45. Fang X, Henao-Mejia J, Henrickson SE. Obesity and immune status in children. Curr Opin Pediatr. 2020; 32 (6): 805-815. doi: 10.1097/MOP.0000000000000953.
46. Painter SD, Ovsyannikova IG, Poland GA. The weight of obesity on the human immune response to vaccination. Vaccine. 2015; 33 (36): 4422-4429.

47. Kelishadi R, Roufarshbaf M, Soheili S, Payghambarzadeh F, Masjedi M. Association of childhood obesity and the immune system: a systematic review of reviews. Child Obes. 2017; 13 (4): 332-346. doi: 10.1089/ chi.2016.0176.

48. Sebastian M, Hsiao CJ, Futch HS, Eisinger RS, Dumeny L, Patel S et al. Obesity and STING1 genotype associate with 23-valent pneumococcal vaccination efficacy. JCI Insight. 2020; 5 (9): e136141. doi: 10.1172/jci. insight.136141.

\section{Financiamiento: AMIP.}

Conflicto de intereses: Ninguno.

Correspondencia:

Enrique Rodríguez Barragán

E-mail: erinfectologo@gmail.com 